\title{
EGFR related mutational status and association to clinical outcome of third-line cetuximab- irinotecan in metastatic colorectal cancer
}

\author{
Karen-Lise G Spindler ${ }^{1 *}$, Niels Pallisgaard ${ }^{2}$, Jan Lindebjerg ${ }^{3}$, Sanne K Frifeldt ${ }^{1}$ and Anders Jakobsen ${ }^{1}$
}

\begin{abstract}
Background: As supplement to KRAS mutational analysis, BRAF and PIK3CA mutations as well as expression of PTEN may account for additional non-responders to anti-EGFR-MoAbs treatment. The aim of the present study was to investigate the utility as biomarkers of these mutations in a uniform cohort of patients with metastatic colorectal cancer treated with third-line cetuximab/irinotecan.

Methods: One-hundred-and-seven patients were prospectively included in the study. Mutational analyses of KRAS, BRAF and PIK3CA were performed on DNA from confirmed malignant tissue using commercially available kits. Loss of PTEN and EGFR was assessed by immunohistochemistry.

Results: DNA was available in 94 patients. The frequency of KRAS, BRAF and PIK3CA mutations were 44\%, 3\% and $14 \%$, respectively. All were non-responders. EGF receptor status by $I H C$ and loss of PTEN failed to show any clinical importance. KRAS and BRAF were mutually exclusive. Supplementing KRAS analysis with BRAF and PIK3CA indentified additional $11 \%$ of non-responders. Patient with any mutation had a high risk of early progression, whereas triple-negative status implied a response rate (RR) of $41 \%(p<0.001)$, a disease control (DC) rate of $73 \%$ $(p<001)$, and a significantly higher PFS of 7.7(5.1-8.6 95\%Cl) versus 2.3 months $(2.1-3.695 \% \mathrm{Cl})(\mathrm{p}<0.000)$.
\end{abstract}

Conclusion: Triple-negative status implied a clear benefit from treatment, and we suggest that patient selection for third-line combination therapy with cetuximab/irinotecan could be based on triple mutational testing.

\section{Background}

Selection of patients for epidermal growth factor receptor targeted monoclonal antibodies (MoAbs) based on tumour KRAS analysis is a major step towards tailored treatment in metastatic colorectal cancer. An increasing amount of data has demonstrated that response to antiEGFR MoAbs is confined to patients with KRAS wild type tumour [1-4]. Patients harbouring KRAS mutations are not likely to benefit from these drugs and KRAS testing is now recommended in this setting [5]. Still, a major fraction of the KRAS wild-type patients are nonresponders, and may not benefit from treatment. The anti-EGFR MoAbs imply a substantial degree of toxicity, and investigations of supplementary predictive factors are therefore highly relevant.

\footnotetext{
* Correspondence: karen-lise.garm.spindler@slb.regionsyddanmark.dk 'Danish Colorectal Cancer Group South, Department of Oncology, Vejle Hospital, Kabbeltoft 25, Vejle, (7100), Denmark

Full list of author information is available at the end of the article
}

Downstream signalling by the RAS-RAF-MAPK pathway is normally very tightly regulated. However, mutations in the KRAS gene render the KRAS protein constitutively active regardless of extracellular EGFR inhibition [2]. Mutations in the genes coding for the other members of the RAS-RAF-MAPK pathway have been identified and may theoretically determine primary resistance to EGFR inhibition in a similar matter $[4,6]$. This has been suggested by cell line studies and clinical data presented by Nicolantonio et al who investigated $B R A F$ and KRAS status in 113 patients treated with panitumumab or cetuximab $[4,7,7]$. Oncogenic activation of BRAF is present in approximately $8-10 \%$ of colorectal tumours and seems mutually exclusive from KRAS mutations according to the literature. A hotspot in colon 600 accounts for the majority of $B R A F$ mutations. However, the relatively low frequency of $B R A S$ mutations detected may be due to poor sensitivity assays (e.g direct sequencing) similar to previously published 
data on KRAS analysis [8]. Consequently, methodological aspects warrant investigation, including development of high-sensitive methods.

Activation of the phosphatidylinositol 3-kinase (PI3K/ $\mathrm{AKT}$ ) pathway is another central mechanism of tumour cell regulation. The PI3K/AKT pathway is critical for development of malignant tumours, constitutes a relevant target for anticancer therapy and regulates the central mTOR pathway, which is influenced by chemotherapeutics and new biological agents $[6,9,10]$. PI3K stimulates the phosphorylation of AKT by interaction with phosphatidylinositol-3-phosphate in the cell membrane. A catalytic subunit of the PI3K is encoded by the PIK3CA gene, which harbour activating mutations in $10-30 \%$ of colorectal tumours according to the literature [6,11-13]. The PI3K/AKT pathway promotes cellular proliferation, invasion, cell survival and neo-angiogenesis $[10,14]$, and PI3K-initiated signalling is normally inhibited by the phosphatase and tensin homolog (PTEN) gene located on chromosome ten. Consequently, PIK3CA mutations and/or loss of PTEN may render malignant cells resistant to EGFR inhibition as described in preclinical studies [15]. This has also been suggested in a recently published clinical trial including 110 patients with metastatic colorectal cancer. A clear association was demonstrated between loss of PTEN/PIK3CA mutations and response to different regimes of EGFR-tageted MoAbs. Furthermore, an independent prognostic value regarding progression free survival was reported by multivariate analysis $[13,16]$.

The EGF receptor expression by IHC has been discharged as predictive marker for response, but the previous data has assessed the receptor status in full populations and not in relation to the mutational status. Just recently a small study has suggested a reconsideration of this aspect by demonstrating a positive predictive value of adding the EGFR expression to KRAS mutational status. The study included 95 patients treated with FOLFIRI or FOLFOX+ cetuximab treatment, and EGFR expression was investigated by IHC and gene expression on primary tumour tissue [17]. The relevance of performing EGF receptor analysis in third-line treatment for KRAS wild-type disease remains to be determined.

As supplement to KRAS analysis, BRAF and PIK3CA mutations as well as loss of PTEN and EGFR expression may account for additional non-responders to EGFR targeted MoAbs. The few previously published studies have combined data from patients treated with different EGFR targeted antibodies as mono- or combinationtherapy in first, second and third-line settings. Various methods for mutational testing have been used. The aim of the present study was to investigate the clinical value of triple KRAS, BRAF and PIK3CA mutational testing by commercially available kits combined with immunohistochemical testing of EGFR and PTEN loss in a material of patients with metastatic colorectal cancer. All patients were treated in a single institution with uniform combination therapy cetuximab-irinotecan (CETIRI).

\section{Methods}

\section{Patient material}

We included 107 heavily pre-treated patients with metastatic colorectal cancer, previously exposed to fluoropyrimidine, irinotecan and oxaliplatin containing regimes and admitted for third line treatment with cetuximab and irinotecan. Clinical eligibility criteria were; Histologically confirmed mCRC (adenocarcinomas) refractory to prior chemotherapy, age over 18 years, ECOG performance status $\leq 2$, adequate organ function, and measurable disease according to the Response Evaluation Criteria in Solid Tumours (RECIST) [18]. Patients were treated with a combination of irinotecan $\left(350 \mathrm{mg} / \mathrm{m}^{2}\right.$ $\mathrm{q} 3 \mathrm{w})$ and cetuximab $\left(400 \mathrm{mg} / \mathrm{m}^{2}\right.$ loading dose followed by weekly $250 \mathrm{mg} / \mathrm{m}^{2}$ ). Response was classified according to RECIST. Treatment was terminated upon progression or in 6 patients after prolonged duration of treatment. Paraffin-embedded primary tumours and/or corresponding metastasis (in one patient only metastatic tissue was available and therefore used for testing) were collected from referring departments of pathological. The study was conducted in accordance with the Danish law after approval by the Regional Ethics Committee. Oral and written consent was obtained from all patients.

\section{DNA purification and KRAS, BRAF and PIK3CA mutational analysis}

DNA was extracted from formalin-fixed paraffinembedded tissue using QIAamp DNA Mini Kit (QIAGEN) after histological confirmation of viable tumour cells on HE stained slides. Mutant KRAS, BRAF and $P I K 3 C A$ were determined by pre-developed kits identifying seven somatic KRAS mutations (G12A, G12R, G12D, G12C, G12S, G12V and G13D), one BRAF mutation (E600V) and 4 PIK3CA mutations (E542K, E545K, E545D and H1047R) (DxS Ltd, Manchester, United Kingdom). Allele-specific real-time quantitative PCR (qPCR) was performed on an ABI7900HT Sequence Detection System (Applied Biosystems, Foster City, CA) according to manufacturer's recommendation.

\section{PTEN and EGFR immunohistochemistry}

Immunohistochmical stainings were performed on $4 \mu \mathrm{m}$ thick sections. Briefly, after deparaffination in graded alcohol solutions antigen retrieval was performed by microwaving in Tris-EGTA buffer, pH 9,0. Subsequently, the slides were incubated with antibody against PTEN (Dako, clone 6H2.1, dilution 1:400), and EGFR 
primary mouse anti-EGFR Mab (EGFR clone $\mathrm{H}-11$, dilution 1:75). The reactions were visualized with Super Sensitive (BioGenex) and ENVISION (DAKO Cytomation-DK) with DAB, followed by counterstaining with haematoxylin. Staining was performed manually.

The PTEN reaction was considered negative if more than $50 \%$ of the tumor cells were negative or weakly stained, compared to the adjacent stromal cells which served as internal control.

EGFR positivity was defined according to DAKO guidelines, any membrane staining above background level was considered positive. Tumours were graded with regard to intensity and amount of membrane staining. A score of staining intensity was assigned as follows: $1+=$ weak $2+=$ moderate and $3+=$ strong membrane staining. The tumour was defined positive if $\geq 1 \%$ of the cells had membranous staining for EGFR according to the DAKO guidelines. A tumour with less than $1 \%$ positive cells was considered EGFR-negative. The score was defined according to the percentage of positively stained tumour cells as follows: $0=$ less than $1 \%, 1=1-10 \%, 2$ $=10-25 \%, 3=25-50 \%, 4=>50 \%$.

Evaluation was independently performed by two investigators (KGS and JL/SKF). The inter- and intra observer reproducibility of assessments of the IHC staining was tested by calculating Cohen's kappa and showed excellent agreement (data not shown).

\section{Statistics}

The association between mutational status and objective response rates, baseline characteristics and skin-toxicity rates was determined by two-tailed Fisher`s exact test. Patients who ended treatment before the first objective tumour evaluating were included in analysis as nonresponders, except from patients experiencing anaphylactic reaction during the first treatment dose. Survival analyses were performed according to the Kaplan-Meier method and survival curves compared by the log-rank test. Progression free survival was defined as time from start of treatment until documented tumour progression or death. Overall survival was calculated from date of first treatment until death of any course. All-p-values were two-sided and considered significant if $\mathrm{p} \leq 0.05$. Statistics were carried out using the NCSS statistical software 2007 v.07.1.5 (NCSS Statistical Software, Utah 84037, USA, http://www.ncss.com).

\section{Results}

One-hundred and seven patients were prospectively included in the study, and 103 patients completed at least one cycle of combination therapy. The median number of cycles was 6 (range 1-18). Eighty-six patients were evaluable for response according to RECIST. Twenty-one patients received less than 3 cycles and were consequently not evaluated by radiological examination according to study schedule. Study treatment was terminated in three cases because of grade 3-4 allergic reactions during the first infusion. One patient withdrew consent after the first dose for psychological reasons. Furthermore 8 patients showed clinical progression and stopped treatment after one cycle and 10 after the second cycle of treatment. Additional patient characteristics are presented in table 1 .

The overall response rate (RR) was 19\% (20/103) and $34 \%(35 / 103)$ achieved stable disease. Eighty-three patients $(81 \%)$ were non-responders as defined by progressive disease $(\mathrm{PD})+$ stable disease $(\mathrm{SD})$ and included 17 patients who showed progression within the first 3 cycles. Skin toxicity data were available in $84 \%$ of the patients and graded according to CTC criteria. At the time of analysis 10 patients were still alive, including 3 patients with stable disease. The median observation time was 7.7 months (range $0.8-31$ ). The median progression free survival of the 103 patients who completed the first cycle was 3.9 months $(2.6-4.795 \% \mathrm{CI})$ and the median overall survival 7.3 months (5.8-9.9 95\%CI).

\section{EGFR expression}

Sixty-eight tumours were available for EGFR staining. Forty-seven percent were negative, whereas 36 (53\%) tumours showed a positive staining including 17 with intensity 2 or greater. EGFR IHC status was not associated with clinicopathological parameters, response or survival in the full cohort (table 2) or in selective analysis in the KRAS wild type patients (data not shown).

\section{Mutational status}

In 13 patients we failed to receive tumour tissue for mutational testing and consequently mutational status was assessed in a total of 94 patients. KRAS mutations were detected in 41 patients (44\%), as presented in table 2. Only 3 patients had BRAF mutations and these were all KRAS wt tumours as show in table 3. A similar mutual exclusiveness was not revealed by the PIK3CA analysis, where 7, 5 and 1 patient harboured mutations located in codon E542, E545 and E1047, respectively. Concomitant PIK3CA and KRAS mutations were observed in 8 patients. Loss of PTEN as defined by less than $50 \%$ of tumour cells positive for PTEN immunostaining was detected in 13 of the $73(18 \%)$ tumours available for PTEN staining.

\section{Mutational profiling and clinico-pathologic characteristics} No significant association was revealed between KRAS, $B R A F$, PIK3CA/PTEN mutational status/loss of staining and age, gender, tumour location or any of the pretreatment characteristics listed in table 1 (Data not shown). 
Table 1 Patient characteristics

\begin{tabular}{lcr}
\hline \multicolumn{1}{c}{ Characteristic } & \multicolumn{2}{c}{ Patients $(\mathbf{n}=\mathbf{1 0 7})$} \\
& No. & (\%) \\
\hline Age, Years & \multicolumn{2}{c}{62} \\
$\quad$ Median & \multicolumn{2}{c}{$38-82$} \\
$\quad$ Range & & \\
\hline Gender & 49 & $(46)$ \\
$\quad$ Female & 58 & $(54)$ \\
$\quad$ Male & & \\
ECOG performance status & 55 & $(51)$ \\
0 & 42 & $(39)$ \\
1 & 10 & $(10)$ \\
2 & &
\end{tabular}

No. of prior chemotherapy regimes for

metastatic disease

\begin{tabular}{lll}
2 & 79 & $(74)$ \\
3 & 27 & $(25)$ \\
NA & 1 & $(1)$ \\
\hline $\begin{array}{l}\text { Primary surgery for CRC } \\
\text { Yes }\end{array}$ & 97 & $(91)$ \\
No & 9 & $(8)$ \\
NA & 1 & $(1)$ \\
\hline Pre-operative chemoradiation & & \\
Yes & 17 & $(16)$ \\
No & 88 & $(82)$ \\
NA & 2 & $(2)$ \\
\hline Anatomic site & & \\
Colon & 40 & $(37)$ \\
Rectosignoideum & 36 & $(34)$ \\
Rectum & 39 & $(36)$ \\
NA & 3 & $(3)$ \\
\hline No. of metastatic sites & & \\
1-2 & & \\
3-5 & 48 & $(45)$ \\
NA & 57 & $(13)$ \\
\hline No & 1 &
\end{tabular}

No of cycles

Median

Range

6

Best response

PR $\quad 20$

SD $\quad 35$

PD 31

Clinical progression* $\quad 17$

NA

Worst Toxicity/Rash grade

$\begin{array}{lll}0 & 15 & (14) \\ 1 & 41 & (39) \\ 2 & 19 & (18) \\ 3 & 11 & (13) \\ \text { NA } & 25 & \text { (23) }\end{array}$

Legend; NA (not assessed), PR, partial response; SD, stable disease, PD progressive disease, ${ }^{*}$ clinical progression (at investigators discretion) in patients who were treated with less than 3 series and consequently not evaluated according to RECIST.

\section{Mutations in KRAS, BRAF or/and PIK3CA/PTEN and clinical} outcome

Clinical outcome and mutational status are presented in table 2 and revealed response rates between $20 \%$ and $37 \%$ in patients tested negative for either mutations.

All three patients with $B R A F$ mutation were non responders and furthermore there was a lower $D C$ rate, median PFS and OS in patients carrying this mutation. The statistical analysis on BRAF mutations did not reach significance, which was most probably due to the low number (3) of patients with BRAF mutations detected.

The PIK3CA mutational analysis revealed a significantly clinical benefit in terms of response, disease control, as well as PFS and overall survival in patients with PIK3CA wild type status. Only this mutation reached statistical significance in univariate overall survival analysis. Patients with PIK3CA mutations achieved a median PFS of 2.3 month and a median OS of 5.8 months compared to 4.6 and 9.2 months, respectively, in patients who did not carry these mutations.

Only one of the 13 patients with loss of PTEN (8\%) achieved response compared to $29 \%$ of patients with normal PTEN expression. The difference was not significant, and even less pronounced with respect to disease control rates in the two groups. Similarly, there was no significant difference in PFS or OS between the two groups. Consequently, this marker was excluded for further analysis, and the KRAS, BRAF and PIK3CA mutations were selected for combined mutational analysis.

\section{Triple mutational status and clinical outcome}

All the 49 patients with KRAS, BRAF or/and PIK3CA mutations were non-responders, and consequently the response rate in patients with triple negative mutational test vas $42 \% \mathrm{p}<0.0001)$. Furthermore, these patients achieved a disease control rate of $73 \%$ (compared to $41 \%$ in the mutational group, $\mathrm{p}=0.001$ ) and the median progression free and overall survival rates were increased to 7.7 and 10.2 months, respectively in this group of patients. Notably, 22\% of the patients with one or more mutations showed early progressed before the third cycle compared to $7 \%$ in the triple negative group $(\mathrm{p}=0.03)$. Kaplan-Meier plots and univariate survival analysis are presented in figure 1.

\section{Discussion}

KRAS mutations only accounts for $30-40 \%$ of the nonresponders to EGFR targeted MoAbs in colorectal cancer. Considering the fact that these agents are now established treatment options in metastatic colorectal cancer and widely used, identification of genetic determinants of primary resistance in KRAS wild type tumour 
Table 2 Outcome according to marker status

\begin{tabular}{|c|c|c|c|c|c|c|}
\hline \multirow[b]{3}{*}{ Total $(n=94)$} & \multirow{3}{*}{$\begin{array}{l}\text { Response } \\
\text { Rate } \\
20 \%\end{array}$} & \multirow{3}{*}{$\begin{array}{l}\text { Disease } \\
\text { Control Rate } \\
56 \%\end{array}$} & \multirow{2}{*}{\multicolumn{2}{|c|}{$\begin{array}{l}\text { Median PFS } \\
\text { months }(95 \% \mathrm{Cl})\end{array}$}} & \multirow{2}{*}{\multicolumn{2}{|c|}{$\begin{array}{l}\text { Median OS } \\
\text { months }(95 \% \mathrm{CI})\end{array}$}} \\
\hline & & & & & & \\
\hline & & & 4.2 & $(2.8-5.1)$ & 8.6 & $(5.9-10.4)$ \\
\hline \multicolumn{7}{|l|}{ KRAS } \\
\hline Mutation $(n=41)$ & $0 \%$ & $45 \%$ & 2.8 & $(2.2-4.1)$ & 6.5 & $(4.8-9.8)$ \\
\hline \multirow[t]{2}{*}{ wild type $(n=53)$} & $37 \%$ & $65 \%$ & 6.5 & $(3.5-8.4)$ & 9,9 & $(6.0-12.2)$ \\
\hline & $p<0.000$ & $p>0.05$ & $p=$ & & $p>$ & \\
\hline \multicolumn{7}{|l|}{ BRAF } \\
\hline Mutation $(\mathrm{n}=3)$ & О\% & $33 \%$ & 2.1 & $(1.9-8.5)$ & 4.5 & $(3.5-25.7)$ \\
\hline \multirow[t]{2}{*}{ Wild type $(n=90)$} & $20 \%$ & $56 \%$ & 4.2 & $(2.8-5.1)$ & 8.6 & $(5.9-10.4)$ \\
\hline & $p>0.05$ & $p>0.05$ & $p>$ & & $p>$ & \\
\hline \multicolumn{7}{|l|}{ PIK3CA } \\
\hline Mutation $(n=13)$ & $0 \%$ & $15 \%$ & 2.2 & $(2.1-2.3)$ & 3.5 & $(3.0-4.7)$ \\
\hline \multirow[t]{2}{*}{ Wild type $(\mathrm{n}=81)$} & $23 \%$ & $63 \%$ & 4.6 & $(3.5-6.2)$ & 9.2 & $(6.6-11.1)$ \\
\hline & $p=0.053$ & $p=0.001$ & $p=$ & & $p=$ & \\
\hline \multicolumn{7}{|l|}{ PTEN IHC } \\
\hline LOSS of PTEN $(n=13)$ & $8 \%$ & $46 \%$ & 3.3 & $(2.1-6.3)$ & 5.9 & $(3.6-10.9)$ \\
\hline \multirow[t]{2}{*}{ Normal expression $(n=59)$} & $23 \%$ & $57 \%$ & 4.4 & $(2.8-6.2)$ & 9.2 & $(6.9-11.1)$ \\
\hline & $p>0.05$ & $p>0.05$ & $p>$ & & $p>$ & \\
\hline \multicolumn{7}{|l|}{ EGFR $॥ \mathrm{HC}$} \\
\hline Positive staining $(n=36)$ & $25 \%$ & $61 \%$ & 4.3 & $(2.2-5.7)$ & 9.0 & $(6.1-10.9)$ \\
\hline \multirow[t]{2}{*}{ Negative staining $(\mathrm{n}=32)$} & $22 \%$ & $43 \%$ & 2.8 & $(2.1-6.9)$ & 8.6 & $(3.5-11.1)$ \\
\hline & $p>0.05$ & $p>0.05$ & $p>$ & & $p>$ & \\
\hline \multicolumn{7}{|l|}{ Triple mutation } \\
\hline${ }^{*}$ mutation $(\mathrm{n}=49)$ & $0 \%$ & $40 \%$ & 2.3 & $(2.1-3.6)$ & 5.8 & $(4.5-9.2)$ \\
\hline \multirow[t]{2}{*}{ Negative $(n=45)$} & $41 \%$ & $73 \%$ & 7.7 & $(5.1-8.6)$ & 10.2 & $(7.1-12.5)$ \\
\hline & $p<0.0000$ & $p=0.001$ & $p<$ & & $p>$ & \\
\hline
\end{tabular}

*Any of the three mutations detected in primary tumour or metastatic tissue.

\# In a few cases DNA was not available for testing of all three mutations.

Response and disease control rates calculated by chi-square test.

Progression free survival (PFS) and overall survival (OS) calculated by log-rank test.

would be essential to further development. The present study investigated relevant mutations related to downstream signalling, which have shown promising results in a few studies.

The purpose of the EGFR analysis was to assess a possible predictive value in KRAS wild type patients only, since most previous data have failed to distinguish between KRAS wt and mutated patients. The EGFR expression by immunohistochemistry was evaluated in 68 patients and did not provide any additional predictive or prognostic value to mutational testing, as suggested by Yen et al [17]. Our findings are supported by the results from a phase III study which investigated chemotherapy and bevasizumab with or without cetuximab (Phase III CAIRO2) as first line treatment for mCRC. EGFR IHC was not a predictive marker in this setting of oxaliplatin based first line regime [19]. Thus, EGFR testing prior to treatment has limited utility, even when accounting for KRAS mutational status.

We confirm the previous literature on KRAS mutational analysis regarding association to non-responsiveness and
PFS, but not OS. Of note, the present frequency of $44 \%$ mutated tumours is marginally higher than previously presented [3,4].

All BRAF mutant tumours were non-responders and all mutually exclusive from mutated KRAS. The overall BRAF V600E mutational rate was $3 \%$ compared to approximately $8-10 \%$ reported in the literature $[2,4,7,12,20-23]$. The low frequency could be a natural statistical variation (The $95 \%$ CIs of $3 / 100$ are $0.6-8.5$ ). Also, the frequency of $B R A F$ mutations is expected to vary between different studies according to criteria for patient selection, line of treatment, PS, limited or advanced disease or further characteristics like geographic variation and microsatellite status amongst others. Furthermore, heterogeneity of $B R A F$ mutational status in tumour compared to metastasis may complicate results. The low frequency of $B R A F$ mutations may also rely on methodological aspects. The method used in our study was therefore validated with a sensitive inhouse PCR method a well as direct sequencing, and the results were in complete agreement (data not shown). 
Table 3 Distribution of the different mutations

\begin{tabular}{|c|c|c|c|}
\hline Patient & KRAS mutations & BRAF mutations & PIK3CA mutations \\
\hline 1 & 12ALA & wt & Wt \\
\hline 2 & 12ALA & wt & Wt \\
\hline 3 & 12ALA & wt & Wt \\
\hline 4 & 12ALA & wt & Wt \\
\hline 5 & $12 A R G$ & wt & Wt \\
\hline 6 & $12 A R G$ & wt & $\mathrm{Wt}$ \\
\hline 7 & $12 \mathrm{ARG}$ & wt & $\mathrm{Wt}$ \\
\hline 8 & $12 \mathrm{ASP}$ & wt & $\mathrm{E} 542 \mathrm{~K}$ \\
\hline 9 & $12 \mathrm{ASP}$ & wt & E542K \\
\hline 10 & $12 \mathrm{ASP}$ & wt & E545K/D** \\
\hline 11 & 12ASP & wt & E545K/D \\
\hline 12 & 12 ASP & wt & E545K/D \\
\hline 13 & 12ASP & wt & Wt \\
\hline 14 & $12 \mathrm{ASP}$ & wt & $W t$ \\
\hline 15 & $12 \mathrm{ASP}$ & wt & Wt \\
\hline 16 & $12 \mathrm{ASP}$ & wt & $\mathrm{Wt}$ \\
\hline 17 & 12 ASP & wt & Wt \\
\hline 18 & $12 \mathrm{ASP}$ & wt & $\mathrm{Wt}$ \\
\hline 19 & $12 \mathrm{ASP}$ & wt & Wt \\
\hline 20 & $12 \mathrm{ASP}$ & wt & $\mathrm{Wt}$ \\
\hline 21 & $12 \mathrm{ASP}$ & wt & Wt \\
\hline 22 & 12ASP & wt & Wt \\
\hline 23 & Mut* & NA & NA \\
\hline 24 & 12 CYS & wt & E542K \\
\hline 25 & $12 \mathrm{CYS}$ & wt & $\mathrm{Wt}$ \\
\hline 26 & 12 CYS & wt & Wt \\
\hline 27 & 12SER & wt & $\mathrm{Wt}$ \\
\hline 28 & 12SER & wt & $\mathrm{Wt}$ \\
\hline 29 & $12 \mathrm{VAL}$ & wt & E542K \\
\hline 30 & $12 \mathrm{VAL}$ & wt & H1047R \\
\hline 31 & $12 \mathrm{VAL}$ & wt & $\mathrm{Wt}$ \\
\hline 32 & $12 \mathrm{VAL}$ & wt & $\mathrm{Wt}$ \\
\hline 33 & $12 \mathrm{VAL}$ & wt & Wt \\
\hline 34 & $12 \mathrm{VAL}$ & wt & Wt \\
\hline 35 & $12 \mathrm{VAL}$ & wt & Wt \\
\hline 36 & 13ASP & wt & $\mathrm{Wt}$ \\
\hline 37 & 13ASP & wt & Wt \\
\hline 38 & $13 \mathrm{ASP}$ & wt & $\mathrm{Wt}$ \\
\hline 39 & 13ASP & wt & Wt \\
\hline 40 & 13ASP & $w t$ & $\mathrm{Wt}$ \\
\hline 41 & 13 CYS & wt & Wt \\
\hline 42 & wt & E600 & Wt \\
\hline 43 & wt & E600 & $W t$ \\
\hline 44 & wt & E600 & Wt \\
\hline 45 & wt & wt & E542K \\
\hline 46 & wt & wt & E542K \\
\hline
\end{tabular}

Table 3 Distribution of the different mutations (Continued)

\begin{tabular}{llll}
\hline 47 & wt & wt & E542K \\
\hline 48 & wt & wt & E545K/D \\
\hline 49 & wt & E545K/D \\
*KRAS mutational status assessed in external institution, but not specified. \\
**The DxS assay does not discriminate between the E545K and E545D \\
mutation.
\end{tabular}

The BRAF analysis accounted for additional 6\% of nonresponders in this study, and is consequently regarded as clinical relevant for further selection, as confirmed by a recent pooled analysis of a larger sample size [4].

The PIK3/PTEN pathway is crucial to EGFR signalling and PTEN evaluation is very central to regulation [10]. Unfortunately the PTEN gene is large and mutations result in a loss of function and therefore not located in hotspots. Consequently, loss of PTEN protein evaluated by immunohistochemistry is a more attractive approach to mutational detection than a complete DNA sequencing. However, the value of PTEN IHC has been debated. In contrast to the studies by Perroni et al and Sartori Bianchi et al $[12,13]$, two other studies failed to demonstrate a significant impact on response by the PTEN assessment $[24,25]$. We aimed to investigate the predictive and prognostic influence of this marker but found it limited in the present material. Another recent study suggested that PTEN IHC was a potential predictive marker when stained on metastatic but not on primary tissue as used in the present study [16]. Methodological difficulties in demonstrating a lack of expression by a semi quantitative method could be an obvious explanation for these findings. Sequencing of the PTEN gene may contribute further to the knowledge. It is however, a complicated and demanding method, and the analysis is probably not justified for use as a clinical tool.

The PIK3CA mutations were detected in 13\% percent of available patients, which is in agreement with the previous literature $[4,6,10,11,13,26]$. $A$ few publications have assessed the clinical impact of these mutations in EGFR targeted therapies. Perrone and colleagues described a significant impact on response and PFS by PIK3CA/ PTEN mutations in a small study of 32 patients [12], and Sartori-Bianchi et al presented a significant PFS and OS survival benefit in non-mutated tumours, and suggested that these mutations may be a rational supplement to KRAS mutational testing with potential for clinical application [13]. However, these results are contradicted by a recent retrospectively study of 200 patients with mCRC, which revealed that 5 of 22 patients carrying PIK3CA mutations responded to treatment, and it was concluded that this mutations do not play a major role in primary resistance to EGFR MoAbs 

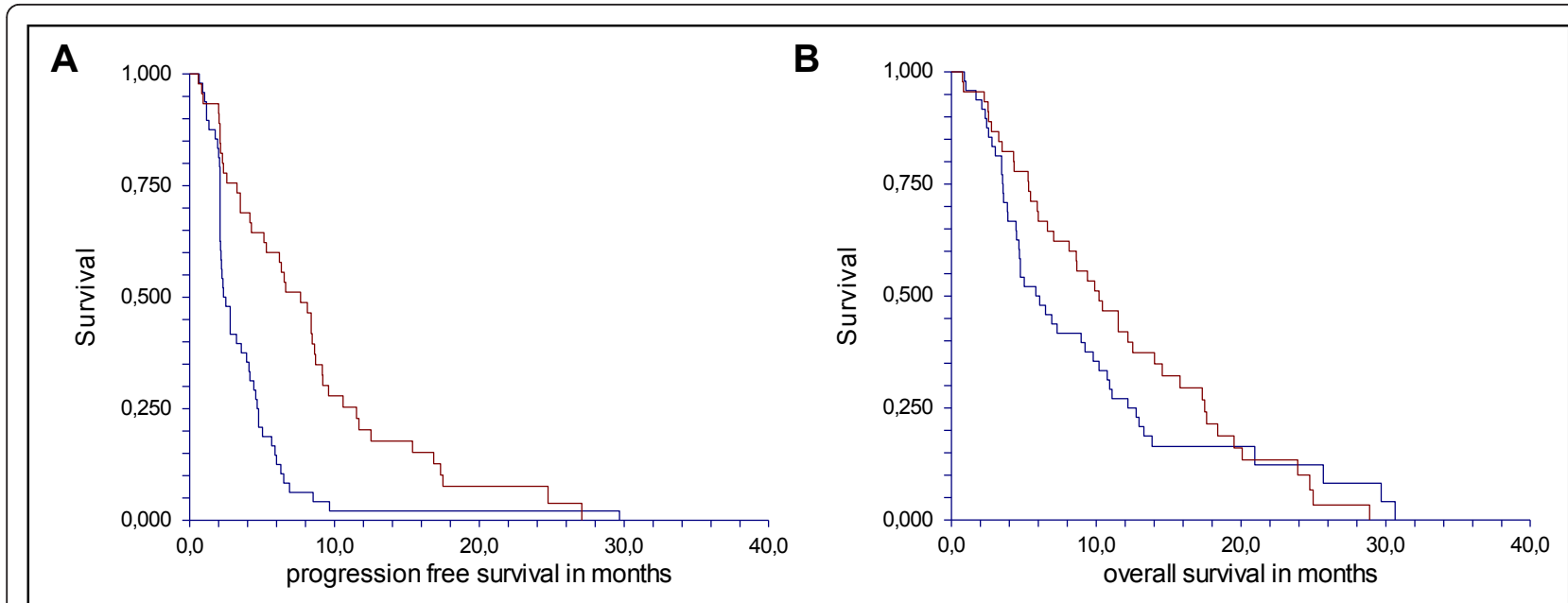

Figure 1 Progression free survival (A) and overall survival (B) in triple negative patients and patients with any mutations detected The median progression free survival was significantly higher in patients with triple negative mutational status (Red: 7.7 months (5.1-8.6, 95\%Cl)) compared to patients with one or more mutations (Blue: 2.3 months $(2.1-3.6,95 \% \mathrm{Cl}) \mathrm{P}<0.000)$. The HR was $2.24(1.45-3.47$ 95\% Cl). The median OS was significantly higher in patients with triple negative mutational status (Red: 10.2 months $(7.1-12.5,95 \% \mathrm{Cl})$ ) compared to patients with one or more mutations (Blue: 5.8 months $(4.5-9.2,95 \% \mathrm{Cl}) \mathrm{p}=0.31)$. The HR was $1.23(0.81-1.8995 \% \mathrm{Cl})$.

[26]. A substantial overlapping between the KRAS and PIK3CA mutations was revealed. Consequently, the PIK3CA analysis was not suggested as a single marker of response. The study was based on a non-homogeneous patients material treated according to 4 different clinical trials with either monotherapy cetuximab or combination therapy, and is therefore not comparable to our patient material. We detected a similarly substantial overlapping between the KRAS and PIK3CA mutations, but all PIK3CA mutated tumours in the present study were non-responders. A recent pooled analysis included data from 1022 patients with mCRC who were treated with EGFR Moabs (according to a variety of treatment regime and lines. The study investigated KRAS, NRAS and $B R A F$ together with different PIK3CA mutations and has confirmed our result, but also suggested a significant different outcome according to the different type of PIK3CA mutations [4]. In the present study all patients with PIK3CA mutations were non-responders, and we were consequently unable to contribute to this aspect.

The sample size of the present study did not allow for comparison of methodological aspects regarding sensitivity for BRAF/PIK3CA mutations, or analysis of potential clinical differences according to the specific locations of the KRAS mutations $[4,16]$. These aspects will need assessment in larger studies. The same applies to development of assays for detection of less frequent KRAS, PIK3CA and BRAF mutations and the clinical implication of these. However, this study included a homogeneous group of patients who received identical treatment dose and schedule in a single institution, in comparison to the majority of recent studies. The observation period allowed for data collection on progression and survival, and revealed a large number of events (97) for survival analysis. Only four patients had not progressed at time of analysis and consequently the strength of statistical analysis on multiple parameters appears acceptable. The median progression free survival in triple negative patients was 7.7 months and held a median overall survival of 10.2 months, indicating a clear benefit from treatment in this group of patients compared to those with one or more mutations. Despite the present limitations, by adding the $B R A F$ and PIK3CA mutational analysis to KRAS testing we identified additional $11 \%$ of the non-responders.

\section{Conclusion}

In conclusion, it is a major challenge to identify patients with a considerable risk of unnecessary toxic side effects and limited benefit from treatment. The present data suggest that patients harbouring any of the three mutations have a very low chance of response and a high risk of early progression before the third cycle of treatment. Furthermore, the triple negative patients achieved a significantly higher disease control rate which translates into a marked increase of progression free survival rate compared to patients with any of the three mutations. Consequently, we suggest that patient selection for third-line combination therapy with EGFR monoclonal antibodies and irinotecan could be based on triple mutational testing.

\section{Acknowledgements}

We thank Dorte Flindt and Birgit Roed Soerensen for technical assistance. 
Funding

Funding was provided from the Research Council at Vejle Hospital and "TRYG-fonden" DK

\section{Author details}

'Danish Colorectal Cancer Group South, Department of Oncology, Vejle Hospital, Kabbeltoft 25, Vejle, (7100), Denmark. ²Danish Colorectal Cancer Group South, Department of Biochemistry, Vejle Hospital, Kabbeltoft 25 Vejle, (7100), Denmark. ${ }^{3}$ Danish Colorectal Cancer Group South, Department of Pathology, Vejle Hospital, Kabbeltoft 25, Vejle, (7100), Denmark.

\section{Authors' contributions}

KGS was responsible for design, concept, protocol writing, implementation, patient inclusion and treatment, immunohistochemical scoring, clinical data collection, data analysis, writing of manuscript and approval. NP implemented and analysed mutational data, participated in manuscript writing and approved of the final manuscript.

UL supervised immunohistochemistry, performed second scoring, participated in writing and approved the final manuscript. SKF treated patients and participated in second scoring of immunohistochemistry, and approved the final manuscript. AJ was responsible for patient treatment, study concept and design and participated in writing and final approval of the manuscript.

\section{Competing interests}

The authors declare that they have no competing interests.

Received: 25 August 2010 Accepted: 25 March 2011

Published: 25 March 2011

\section{References}

1. Amado RG, Wolf M, Peeters M, Van CE, Siena S, Freeman DJ, et al: Wildtype KRAS is required for panitumumab efficacy in patients with metastatic colorectal cancer. J Clin Oncol 2008, 26:1626-1634.

2. Benvenuti S, Sartore-Bianchi A, Di NF, Zanon C, Moroni M, Veronese S, et al: Oncogenic activation of the RAS/RAF signaling pathway impairs the response of metastatic colorectal cancers to anti-epidermal growth factor receptor antibody therapies. Cancer Res 2007, 67:2643-2648.

3. Linardou H, Dahabreh IJ, Kanaloupiti D, Siannis F, Bafaloukos D, Kosmidis P, et al: Assessment of somatic k-RAS mutations as a mechanism associated with resistance to EGFR-targeted agents: a systematic review and meta-analysis of studies in advanced non-small-cell lung cancer and metastatic colorectal cancer. Lancet Oncol 2008, 9:962-972.

4. De Roock W, Claes B, Bernasconi D, De SJ, Biesmans B, Fountzilas G, et al: Effects of KRAS, BRAF, NRAS, and PIK3CA mutations on the efficacy of cetuximab plus chemotherapy in chemotherapy-refractory metastatic colorectal cancer: a retrospective consortium analysis. Lancet Oncol 2010, 11:753-762.

5. Allegra CJ, Jessup JM, Somerfield MR, Hamilton SR, Hammond EH, Hayes DF, et al: American Society of Clinical Oncology provisional clinical opinion: testing for KRAS gene mutations in patients with metastatic colorectal carcinoma to predict response to anti-epidermal growth factor receptor monoclonal antibody therapy. J Clin Oncol 2009, 27:2091-2096.

6. Barault L, Veyrie N, Jooste V, Lecorre D, Chapusot C, Ferraz JM, et al: Mutations in the RAS-MAPK, PI(3)K (phosphatidylinositol-3-OH kinase) signaling network correlate with poor survival in a population-based series of colon cancers. Int J Cancer 2008, 122:2255-2259.

7. Di Nicolantonio F, Martini M, Molinari F, Sartore-Bianchi A, Arena S, Saletti P, et al: Wild-type BRAF is required for response to panitumumab or cetuximab in metastatic colorectal cancer. J Clin Oncol 2008, 26:5705-5712.

8. Garm Spindler KL, Pallisgaard N, Rasmussen AA, Lindebjerg J, Andersen RF, Cruger D, et al: The importance of KRAS mutations and EGF61A > G polymorphism to the effect of cetuximab and irinotecan in metastatic colorectal cancer. Ann Oncol 2009, 20:879-884.

9. bdul-Ghani R, Serra V, Gyorffy B, Jurchott K, Solf A, Dietel M, et al: The PI3K inhibitor LY294002 blocks drug export from resistant colon carcinoma cells overexpressing MRP1. Oncogene 2006, 25:1743-1752.

10. Samuels Y, Diaz LA Jr, Schmidt-Kittler O, Cummins JM, Delong L, Cheong I, et al: Mutant PIK3CA promotes cell growth and invasion of human cancer cells. Cancer Cell 2005, 7:561-573.
11. Ogino S, Nosho K, Kirkner GJ, Shima K, Irahara N, Kure S, et al: PIK3CA mutation is associated with poor prognosis among patients with curatively resected colon cancer. J Clin Oncol 2009, 27:1477-1484.

12. Perrone F, Lampis A, Orsenigo M, Di BM, Gevorgyan A, Losa M, et al: PI3KCA/PTEN deregulation contributes to impaired responses to cetuximab in metastatic colorectal cancer patients. Ann Oncol 2009, 20:84-90.

13. Sartore-Bianchi A, Martini M, Molinari F, Veronese S, Nichelatti M, Artale S, et al: PIK3CA mutations in colorectal cancer are associated with clinical resistance to EGFR-targeted monoclonal antibodies. Cancer Res 2009, 69:1851-1857.

14. Samuels Y, Diaz LA Jr, Schmidt-Kittler O, Cummins JM, Delong L, Cheong I, et al: Mutant PIK3CA promotes cell growth and invasion of human cancer cells. Cancer Cell 2005, 7:561-573.

15. Jhawer M, Goel S, Wilson AJ, Montagna C, Ling YH, Byun DS, et al: PIK3CA mutation/PTEN expression status predicts response of colon cancer cells to the epidermal growth factor receptor inhibitor cetuximab. Cancer Res 2008, 68:1953-1961.

16. Loupakis F, Pollina L, Stasi I, Ruzzo A, Scartozzi M, Santini D, et al: PTEN expression and KRAS mutations on primary tumors and metastases in the prediction of benefit from cetuximab plus irinotecan for patients with metastatic colorectal cancer. J Clin Oncol 2009, 27:2622-2629.

17. Yen LC, Uen YH, Wu DC, Lu CY, Yu FJ, Wu IC, et al: Activating KRAS mutations and overexpression of epidermal growth factor receptor as independent predictors in metastatic colorectal cancer patients treated with cetuximab. Ann Surg 2010, 251:254-260.

18. Therasse P, Arbuck SG, Eisenhauer EA, Wanders J, Kaplan RS, Rubinstein L, et al: New guidelines to evaluate the response to treatment in solid tumors. European Organization for Research and Treatment of Cancer, National Cancer Institute of the United States, National Cancer Institute of Canada. J Natl Cancer Inst 2000, 92:205-216.

19. Tol J, Dijkstra JR, Klomp M, Teerenstra S, Dommerholt M, Vink-Borger ME, et al: Markers for EGFR pathway activation as predictor of outcome in metastatic colorectal cancer patients treated with or without cetuximab. Eur J Cancer 2010, 46:1997-2009.

20. Cappuzzo F, Finocchiaro G, Rossi E, Janne PA, Carnaghi C, Calandri C, et al: EGFR FISH assay predicts for response to cetuximab in chemotherapy refractory colorectal cancer patients. Ann Oncol 2008, 19:717-723.

21. De Roock W, Piessevaux H, De SJ, Janssens $M$, De HG, Personeni $N$, et al: KRAS wild-type state predicts survival and is associated to early radiological response in metastatic colorectal cancer treated with cetuximab. Ann Oncol 2008, 19:508-515.

22. Lievre $A$, Bachet JB, Le CD, Boige $V$, Landi B, Emile JF, et al: KRAS mutation status is predictive of response to cetuximab therapy in colorectal cancer. Cancer Res 2006, 66:3992-3995.

23. Moroni M, Veronese S, Benvenuti S, Marrapese G, Sartore-Bianchi A, Di NF, et al: Gene copy number for epidermal growth factor receptor (EGFR) and clinical response to antiEGFR treatment in colorectal cancer: a cohort study. Lancet Oncol 2005, 6:279-286.

24. Razis E, Briasoulis E, Vrettou E, Skarlos DV, Papamichael D, Kostopoulos I, et al: Potential value of PTEN in predicting cetuximab response in colorectal cancer: an exploratory study. BMC Cancer 2008, 8:234.

25. Laurent-Puig P, Cayre A, Manceau G, Buc E, Bachet JB, Lecomte T, et al: Analysis of PTEN, BRAF, and EGFR status in determining benefit from cetuximab therapy in wild-type KRAS metastatic colon cancer. J Clin Oncol 2009, 27:5924-5930.

26. Prenen H, De SJ, Jacobs B, De RW, Biesmans B, Claes B, et al: PIK3CA mutations are not a major determinant of resistance to the epidermal growth factor receptor inhibitor cetuximab in metastatic colorectal cancer. Clin Cancer Res 2009, 15:3184-3188.

\section{Pre-publication history}

The pre-publication history for this paper can be accessed here: http://www.biomedcentral.com/1471-2407/11/107/prepub

doi:10.1186/1471-2407-11-107

Cite this article as: Spindler et al:: EGFR related mutational status and association to clinical outcome of third-line cetuximab-irinotecan in metastatic colorectal cancer. BMC Cancer 2011 11:107. 